\title{
Calculation of absolute diffusion rates in oxides
}

\author{
M J L Sangster $\div$ and A M Stoneham \\ $\div$ J J Thomson Physical Laboratory, University of Reading. Whiteknights. Reading. UK

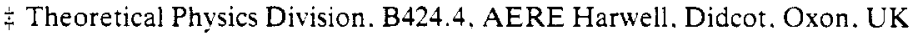

Received 8 June 1984

\begin{abstract}
We have calculated the absolute rate of diffusion for $\mathrm{Mg}^{2-}$ and $\mathrm{Fe}^{3-}$ ions in $\mathrm{MgO}$ using atomistic modelling. Our calculations use a shell model, incorporating tested interatomic potentials, and exploit recent advances in computer codes. The agreement is extremely good where experimental data are available for comparison. For $\mathrm{Mg}^{2+}$ in $\mathrm{MgO}$ at $1400^{\circ} \mathrm{C}$ we predict a pre-exponential factor of $32.9 \mathrm{THz}$ using simple Vineyard theory (experiment $18 \pm 7 \mathrm{THz}$ after correction for the important volume dependence of the activation energy) and an activation energy of $2.26 \mathrm{eV}$ (experiment $2.3 \pm 0.2 \mathrm{eV}$ ). Close inspection of the energy changes for displacements from the saddle point normal to the jump path shows that within $k T$ of the saddle point energy there are significant departures from the harmonic dependence required for validity of Vineyard theory. Corrections by both analytical methods and numerical integration improve agreement with experiment. predicting 23-25 THz overall. For $\mathrm{Fe}^{3-}$ much slower diffusion is predicted even though the jump path bifurcates to give two saddle points. We do not predict the rapid $\mathrm{Fe}^{3-}$ motion reported in aggregation experiments and conclude that other mechanisms are involved.

We have also used the dynamical theory of Rice and Slater which gives similar. but by no means identical, predictions for the diffusion rates.
\end{abstract}

\section{Introduction}

Our paper is concerned with the calculation of diffusion rates in crystalline solids. Its aim is the prediction of absolute rates, not simply activation energies, by the best current methods and computer modelling. This aim reflects two recent developments in transport studies. The first is theoretical, and relates to the recent codes available for the prediction of harmonic vibrations of distorted crystals. This topic is described in $\S 2$. The second development is experimental. In diffusion studies of ceramics like $\mathrm{MgO}$ (see Wuensch 1982) there are no clear regimes identifiable as extrinsic and intrinsic. Thus, only with the recent mobility data (Sempolinski and Kingery 1980) can one obtain unambiguous jump frequency data to compare with our calculations. We have also been encouraged by the increased emphasis on absolute jump rates in a variety of ionic conductors. Sometimes unexpectedly high rates are reported (e.g. Almond and West 1983), sometimes unexpectedly low rates (e.g. Huberman and Boyce 1978). In other cases, ionic diffusion is only one component of a solid state process (e.g. Weeks $e$ t al 1980 ) and knowledge of the jump frequency itself is of value.

We calculate here jump frequencies for $\mathrm{Mg}^{2+}$ vacancies and for vacancy-substitutional $\mathrm{Fe}^{3+}$ exchange in $\mathrm{MgO}$. Much of the background to our work and some related 
calculations are discussed in our previous paper (Sangster et al 1984) which is extended in a number of respects in the present paper.

\section{Calculations of jump frequencies}

The prediction of jump frequencies involves three components. The first concerns the statistical mechanics relating the jump frequency to the energy surfaces (i.e. the total energy as a function of the ionic positions), the masses of the particles, and temperature. Here we shall follow mainly the formulation of Vineyard (1957) based on reaction rate theory, though we shall also cite results from the dynamical theory of Rice (1958) and Slater (1959). The second component concerns the calculation of the energy surfaces, achieved by standard methods. Since we are interested in jump frequencies, more detailed knowledge of the energy surface is required than for a mere estimate of activation energy. The third strand in the calculation is the harmonic lattice dynamics for various stages of the jump process.

\subsection{Reaction rate theory and dynamical theory}

In this section we discuss the relationship between energy surfaces, assumed known, and jump rates. We shall concentrate on the way the jump rates can be obtained in practice; the considerable points of principle are discussed elsewhere (Flynn 1972, Sangster et al 1984).

Reaction rate theory (Glasstone et al 1941) makes direct use of equilibrium statistical mechanics. In his development of the theory for applications to solids, Vineyard (1957) adopts a many-body normal mode approach, and identifies crossings between equilibrium configurations with the passage through zero of the coordinate of the normal mode which is unstable at the saddle point. Making the assumption that the energy for configurations around the saddle point may be adequately represented within the harmonic approximation, he arrives at the following expression for the pre-exponential frequency factor:

$$
\nu_{0}=\left(\prod_{i=1}^{3 N} \nu_{i}\right) /\left(\prod_{i=1}^{3 N-1} \nu_{j}\right)
$$

The numerator is the product of the normal mode frequencies, $\nu_{i}$, at the equilibrium configuration, and the denominator is the product of the frequencies, $\nu_{i}^{\prime}$, of the stable normal modes at the saddle point configuration; $N$ is the number of atoms in the crystal.

We shall use (1) directly, using frequencies calculated by the methods of $\S 2.3$. However, we shall also need a simple generalisation of (1) since the harmonic expansion is not sufficient in certain cases. The harmonic expansion is exploited by Vineyard in two separate ways. The first is to factorise integrals $I_{\mathrm{S}}$ at the saddle point and $I_{\mathrm{A}}$ at the equilibrium configuration into products of simpler integrals, one for each mode. The second use is to ensure that these simpler component integrals all have an exactly soluble form, specifically

$$
I^{(i)}=\int_{-\infty}^{\infty} \mathrm{d} x_{i} \exp \left(-\delta \varphi_{i} / k T\right)
$$

where $\delta \varphi_{i}$ is the energy change due to a displacement $x_{i}$ from the relevant minimum; for 
a harmonic system, $\delta \varphi_{i}$ is proportional to $x_{i}^{2}$. In $\S 2.2$ we present calculations of energy surfaces around the saddle points and find that $\delta \varphi_{i}$ is significantly non-harmonic while within $k T$ of the saddle point energy. In $\S 3.3$ we shall estimate (by numerical integration) the correction factor to the saddle point integral for $\mathrm{Mg}$ in $\mathrm{MgO}$ which arises from the hardening of the potential with increasing displacement from the saddle point. It may be helpful to consider an extreme example analytically. Suppose that for one coordinate there are infinite barriers at $x= \pm X$, the potential behaving harmonically for $|x| \leqslant X$ and all other coordinates being harmonic. There is then a correction factor $\operatorname{erf}\left[\left(\frac{1}{2} M \omega^{2} X^{2} / k T\right)^{1 / 2}\right]$ to the Vineyard result, where $\frac{1}{2} M \omega^{2} X^{2}$ is just the strain energy at $x= \pm X$. At $1400^{\circ} \mathrm{C}$. $k T$ is $0.1441 \mathrm{eV}$; for a strain energy of $0.1 \mathrm{eV}$. the Vineyard prediction is reduced by a factor 0.76 .

In the dynamical theory of Slater (1958) and Rice (1959) the jump rate is determined by motion in a reaction coordinate. The simplest choice of reaction coordinate involves only motion of the diffusing atom from its normal site towards the saddle point. More realistic choices involve accompanying adjustments of the positions and polarisations of nearby ions. In each of these situations, the jump rate can be estimated from the $\nu_{i}$ of (1) or from the energy surfaces near the equilibrium sites. One of our examples $\left(\mathrm{MgO}: \mathrm{Fe}^{3+}\right)$ presents problems of principle, however, since the jump path bifurcates near the saddle point. There are thus ambiguities in choosing the reaction coordinate, for the paths which, when extrapolated, take the diffusing particle to the saddle point are not the lowest-energy paths near the equilibrium geometry.

\subsection{Energy surfaces}

We have investigated cation vacancy jump rates in pure $\mathrm{MgO}$ and in $\mathrm{MgO}$ doped with $\mathrm{Fe}^{3-}$. In the former case the vacancy jump can be considered as an interchange of a vacant site on the cation sublattice and a nearest-neighbour $\mathrm{Mg}^{2+}$ ion, with a saddle point configuration consisting of two adjacent vacant sites and an interstitial $\mathrm{Mg}^{2+}$ ion equidistant from the vacancies. In the $\mathrm{MgO}: \mathrm{Fe}^{3+}$ case we consider the jump when the mobile nearest neighbour is one of the $\mathrm{Fe}^{3+}$ impurities.

Figure 1 shows energy contours for fully relaxed configurations with the jumping ion at positions in the bisecting plane normal to the line joining initial and final sites (taken as $\langle 110\rangle)$. These results were calculated with interionic potentials obtained earlier by us (Sangster and Stoneham 1981) using the HADEs (Norgett 1974. 1977) package to minimise the configuration energy subject to the constraint of holding the jumping ion at chosen positions in the plane. It can be seen that for the $\mathrm{Mg}^{2+}$ ion jump (figure $1 a$ ) the saddle point is at the midpoint of the line between the vacant sites, i.e. at the point of minimum energy in the plane shown in the plot. The activation energy for the jump, that is the difference in energy between this saddle point configuration and the isolated vacancy configuration, is calculated as $2.2 \mathrm{eV}$ when we use the room-temperature lattice parameter for $\mathrm{MgO}\left(a_{0}=2.106 \AA\right)$ and $1.9 \mathrm{eV}$ when we take $a_{0}=2.148 \AA$, estimated from the experimental lattice expansion to correspond to a temperature of $1400^{\circ} \mathrm{C}$. The difference in activation energies may be re-expressed as an activation volume $\Omega$, which can be estimated (Gillan 1981) from $\Omega=-\frac{1}{3} \kappa a_{0}\left(\Delta E_{\text {activation }} / \Delta a\right)$ with $K$ the model isothermal compressibility $\left(4.36 \times 10^{-12} \mathrm{~Pa}^{-1}\right.$ from the elastic constants quoted by Sangster and Stoneham (1981)). We obtain $\Omega=0.37 a_{0}^{3}$. Sempolinski and Kingery (1980) have estimated the mobility of $\mathrm{Mg}^{2+}$ vacancies in $\mathrm{MgO}$ from their measurements of the temperature dependence of the ionic conductivity. The value for the activation energy given by Sempolinski and Kingery from their measurements in the temperature range 


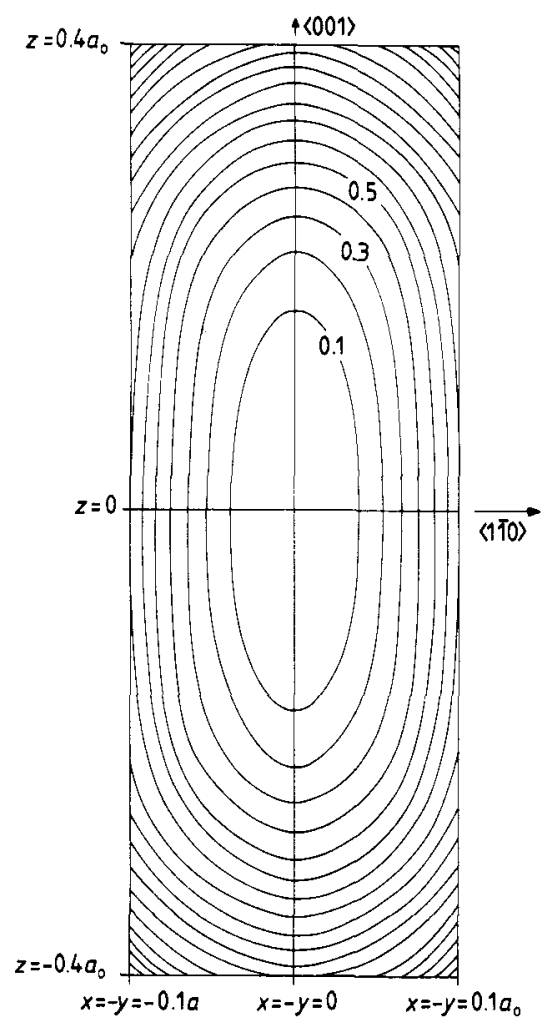

(a)

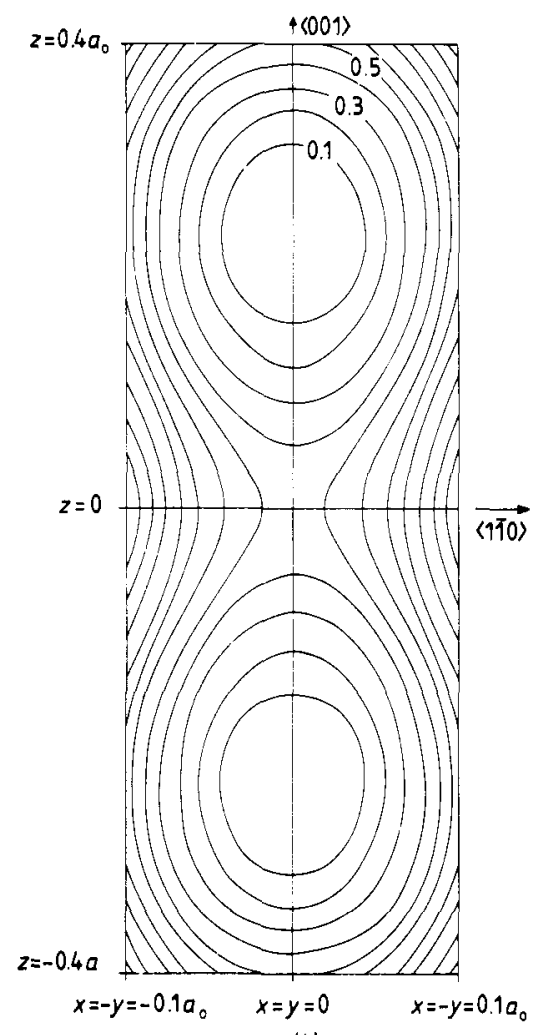

(b)

Figure 1. Energy contours in the saddle point planes for $(a) \mathrm{Mg}^{2+}$ diffusion in $\mathrm{MgO}$ and (b) $\mathrm{Fe}^{3+}$ diffusion in $\mathrm{MgO}$. The jump direction is normal to the page $((110\rangle)$ and the two oxygen ions straddling the jump path are along the $\langle 1 \overline{1} 0\rangle$ direction. Energies are plotted at $0.1 \mathrm{eV}$ intervals and are relative to the saddle point energy. The energy at a point in the plane is found by constraining the diffusing ion at the point and allowing full relaxation of neighbouring ions.

$1200-1600^{\circ} \mathrm{C}$ is $2.3 \pm 0.2 \mathrm{eV}$, close to our predictions extrapolated to low temperature. We shall discuss the significance of this later.

The higher curvature in the $\langle 110\rangle$ direction is to be expected from the proximity of $\mathrm{O}^{2-}$ neighbours. In the $\langle 001\rangle$ direction the curvature is low and, when the $\mathrm{Mg}^{2+}$ ion is replaced by an $\mathrm{Fe}^{3+}$ ion (figure $1 b$ ), the central position becomes a maximum for the $\langle 001\rangle$ direction. Bifurcated minimum-energy jump paths result with the saddle points displaced from the central position by $\pm 0.238 a_{0}$ along the $\langle 001\rangle$ direction. Our calculations give the activation energy for the $\mathrm{Fe}^{3+}$ ion jump as $2.7 \mathrm{eV}$, with the energy of the saddle point configurations $0.48 \mathrm{eV}$ below that of the symmetric (midpoint) configuration. For the $\mathrm{Fe}^{3+}$ jump there are no results similar to those for $\mathrm{Mg}^{2+}$ for comparison with our calculations although $\mathrm{MgO}: \mathrm{Fe}^{3+}$ was studied in the above work by Sempolinski and Kingery and the formation energies of $\mathrm{Fe}^{3+}$-vacancy pair configurations in $\mathrm{MgO}$ have been examined by Gourdin et al (1979). The review by Wuensch (1983) quotes activation energies of around $1.8 \mathrm{eV}$ for $\mathrm{Fe}$ (arguably $\mathrm{Fe}^{2+}$ not $\mathrm{Fe}^{3+}$ ) in $\mathrm{MgO}$. The value for the activation energy found from tracer diffusion experiments by Blank and Pask (1969) is $3.2 \mathrm{eV}$. The agreement between this value and our calculated energy difference of $3.18 \mathrm{eV}$ between the equilibrium and midpoint configurations is almost certainly coincidental. 


\subsection{Harmonic frequencies}

2.3.1. Estimate from energy surfaces using the dynamical theory. In the dynamical theory the motion of the diffusing ion is described by a reaction coordinate, not a normal mode. The pre-exponential frequency factor, often described as the attempt frequency, is proportional to the square root of the curvature of the energy surface at the equilibrium configuration in the direction of the reaction coordinate. Both this curvature and the effective mass associated with the reaction coordinate depend on whether only the shells (electronic polarisation alone) or both shells and cores (ionic and electronic polarisation) are able to follow the motion of the diffusing ion. Results for the effective frequencies for dynamical theory in these two limits are given in table 1. In all cases we have taken the mass associated with the reaction coordinate to be that of only the diffusing ion: for the results which include core relaxations the effective mass should be increased and consequently the effective frequency reduced. It is assumed that the motion is directly towards the final site and the results do not include the factor 2 for $\mathrm{Fe}^{3+}$ or any other aspect of the bifurcation in that case.

Table 1. Estimates of effective frequencies (THz) for the dynamical theory. These values are obtained from curvatures of energy surfaces assuming the mass associated with the reaction coordinate to be that of the diffusing ion alone.

\begin{tabular}{lll}
\hline $\begin{array}{l}\text { Diffusing } \\
\text { ion }\end{array}$ & $\begin{array}{l}\text { Allowing relaxation } \\
\text { of shells and cores }\end{array}$ & $\begin{array}{l}\text { Allowing shell } \\
\text { relaxations only }\end{array}$ \\
\hline $\mathrm{Mg}^{2-}$ & 8.24 & 10.70 \\
$\mathrm{Ca}^{2+}$ & 6.14 & 8.64 \\
$\mathrm{Mn}^{2-}$ & 5.65 & 7.12 \\
$\mathrm{Fe}^{2-}$ & 5.55 & 6.98 \\
$\mathrm{Fe}^{3+}$ & 5.92 & 8.48 \\
$\mathrm{Co}^{2+}$ & 5.78 & 7.39 \\
$\mathrm{Ni}^{2-}$ & 5.72 & 7.15 \\
\hline
\end{tabular}

The results show two significant features. First, the dynamical theory gives rather low frequencies, well below the value $15.2 \mathrm{THz}\left((3 / 5)^{1 / 2} \nu_{\text {Debye }}\right.$; see Flynn (1972)) often suggested. This discrepancy is enhanced if we allow for the increase in effective mass associated with motion of neighbouring ions. Secondly, the relative frequencies decrease in order $\mathrm{Mg}^{2+}>\mathrm{Ca}^{2+}>\mathrm{Fe}^{3+}>\left(\mathrm{Mn}^{2+}, \mathrm{Fe}^{2+}, \mathrm{Co}^{2+}, \mathrm{Ni}^{2+}\right)$, with these divalent transition metals having essentially the same frequencies. Wuensch's (1983) review of experimental diffusion data also suggests that $\mathrm{Mg}^{2+}$ has a higher frequency than $\mathrm{Ca}^{2+}:$ it is hard to draw specific conclusions about the other cases. Since there are ambiguities in the precise application of the dynamical theory (both concerning the relaxation of other ion cores and in relation to bifurcating paths) we feel that it is mainly of qualitative value where its advantage is relative simplicity.

2.3.2. Supercell methods. Recently some solutions to problems involving the dynamics of defects in crystals have been found by using the 'supercell' method rather than the more usual Green function techniques. In the supercell method a large periodically repeated unit cell containing the defects under consideration (e.g. impurities, vacancies, interstitials, etc) and with neighbouring atoms polarised and relaxed to equilibrium is 
set up. With the computational power now readily available it is then a straightforward matter to calculate from interaction potential models the eigenfrequencies and vectors for normal modes of the system, since translational symmetry is restored and the usual methods for perfect lattices can be applied, albeit with the large number of atoms in the unit cell. The relaxations of neighbouring atoms, including their polarisations, must first be calculated from the same interaction potential models and for this two fairly standard procedures are possible: either the defect system can be embedded in an infinite and otherwise perfect crystal and the relaxations calculated using programmes such as HADES or, more consistently, relaxations in the periodic structure can be calculated with codes such as PLUTO (Catlow and Norgett 1976). An inconvenience of the former method is that some adjustments to the positions and polarisations of atoms on or near cell boundaries are required to enforce periodicity.

Applications of the supercell method include calculations of vibrational entropies of defects in crystals (Harding and Stoneham 1980, Sangster et al 1984), an investigation of electron-hole excitation in $\mathrm{UO}_{2}$ (Harding et al 1980) and a study of the infrared absorption due to off-centre $\mathrm{Li}$ in $\mathrm{KCl}$ (Sangster and Stoneham 1982). These problems can of course be tackled by Green function methods: see Jacobs and Gillan (1983) for a discussion of the Green function method applied to vibrational entropy problems. In such Green function approaches force constant changes must be calculated from the model potentials in a manner which is consistent with the type of Green functions used. Almost invariably these functions relate to ionic core displacements, with polarisation terms, such as shell displacements in the shell model, having been eliminated using the adiabatic approximation. This being the case, the corresponding effective force constant changes must be calculated under the same approximation. As Sangster and Rowell $(1982,1983)$ have shown, such calculations can be performed routinely, but their results indicate that the defect space often has to be extended to quite distant neighbours of the foreign ions and defects. Furthermore, in cases such as off-centre $\mathrm{Li}$ in $\mathrm{KCl}$ mentioned above, the lower point group symmetry leads to a considerable increase in the number of Green function terms required. Both these considerations reduce the attractions of the Green function approach. Furthermore, if the defect carries a net charge it is not clear how the usual formalism for changes of mass and force constant should be extended. Finally, there is an inherent inconsistency in treating the dynamics of the host crystal with a polarisable-ion model (such as the shell model) and then expressing a defect problem in terms of only ion Green functions. (There have been a few attempts at treating the defect consistently, notably by Page and Strauch (1967) who have included core-core, core-shell and shell-shell Green functions in their analysis of the infrared absorption due to U-centres in alkali halides, but such treatments are exceptional.) Thus, while the existence of standard packages and sufficient computational power to implement these may appear a poor reason for taking the apparently retrogressive step of rejecting the elegance of Green function methods, it does appear worthwhile to exploit the alternative direct methods, such as the supercell method, which treat the dynamics of defective lattices on the same footing as that of perfect host lattices.

The main technical problem with the supercell method is that of storage for the large matrices required. In the previous work referred to above an existing lattice dynamics package was used and this meant that, with a restriction to 1.5 Mbyte of store, only cells containing up to 24 atoms could be considered. At this level the artificially imposed periodicity of the defect structure is likely to produce spurious effects. Along with D K Rowell we have now produced a version of the program which, without degrading the efficiency, reduces the storage requirements by a factor of around five. Supercell cal- 
culations with up to about 100 atoms per cell become feasible; our results reported in the next section are for calculations with 64 atomic sites per cell.

\section{Predictions of absolute rates}

\subsection{Results of Vineyard theory}

In this section we discuss the results of our calculations using reaction rate theory. We have estimated the ratio of products in equation (1) for the two cases under consideration by calculating the normal mode frequencies for 63-atom supercells in relaxed equilibrium and saddle point configurations. For the saddle point configurations we obtain one imaginary frequency as required. We carried through the calculations using only one $q$-vector in each case, taking a vector just sufficiently displaced from the Brillouin zone centre to give Lyddane-Sachs-Teller splittings. This simplifying approximation, which has been used and checked in analogous electronic calculations (see, for example, Evarestov and Lovchikov 1979), relies on the effective averaging over the zone which results from the folding back of the phonon branches in the reciprocal lattice of the supercell. Substitution of the calculated frequencies gives

$$
\nu_{0}=32.91 \mathrm{THz} \text { for the } \mathrm{Mg}^{2+} \text { jump }
$$

and

$$
\nu_{0}=1.21 \mathrm{THz} \text { for the } \mathrm{Fe}^{3+} \text { jump. }
$$

Since in the case of the $\mathrm{Fe}^{3+}$ jump there is a pair of saddle points, the second frequency should be doubled for use in expressions for jump rates.

Compared with typical lattice frequencies ( $\leqslant 20 \mathrm{THz}$ for $\mathrm{MgO}$ ) the Vineyard expression gives an anomalously high frequency in the first case and an anomalously low frequency in the second. It is easy to see that the form of equation (1) can give rise to either type of 'anomaly'; all that is involved is the ratio of curvatures at the equilibrium and saddle points. For the $\mathrm{Mg}^{2+}$ jump the ratio of the curvatures along the $\langle 001\rangle$ direction for the energy surfaces (figure 1(a) and a corresponding surface around the equilibrium configuration) is $(3.6)^{2}$ and hence, if we associate the same mass with $\langle 001\rangle$ displacements from both equilibrium and saddle point configurations, the frequency ratio is 3.6 . As confirmation, examination of our sample of normal mode frequencies shows that the high value for $\nu_{0}$ arises principally from a low-frequency $(2.04 \mathrm{THz})$ mode at the saddle point. The eigenvector for this mode is dominated by the $\langle 001\rangle$ displacement of the jumping $\mathrm{Mg}^{2+}$ ion.

\subsection{Experimental results for $\mathrm{Mg}^{2+}$ motion}

Jump frequencies are generally expressed as

$$
\Gamma=\Gamma_{0} \exp \left(-E_{\mathrm{A}} / k T\right)
$$

where $E_{\mathrm{A}}$ is the activation energy for the jump process and where evaluation of the premultiplying factor $\Gamma_{0}$ is required if absolute rates are to be determined. Sempolinski and Kingery (1980) deduce from their measurements that the mobility for vacancy motion in $\mathrm{MgO}$ is

$$
\mu=(8800 / T) \exp (-2.2 \mathrm{eV} / k T) \mathrm{cm}^{2} \mathrm{~V}^{-1} \mathrm{~s}^{-1}
$$


The mobility is related to the jump frequency $\Gamma$ for a specific jump (see, for example, Lidiard 1957) by

$$
\mu=\frac{1}{3} \Gamma N a^{2} e / k T
$$

where $N$ is the number of equivalent jumps of length $a$. In our case $N=12$ and $a=$ $\sqrt{2} a_{0}$. Thus from the experimental results we obtain for a specific jump $\Gamma_{0}=$ $210 \pm 80 \mathrm{THz}$, where the uncertainty corresponds with Sempolinski and Kingery's quoted error in the diffusion constant (which is related to the mobility through the Nernst-Einstein relation). In our earlier paper (Sangster et al 1984) the factor $N$ in equation (4) was incorrectly omitted in our analysis.

An immediate cause for concern with this estimate is that it is an order of magnitude larger than the maximum lattice frequency in $\mathrm{MgO}$ (around $20 \mathrm{THz}$ ). Intuitively one might expect this host frequency to give a rough upper bound to the pre-exponential frequency factor. We believe that the interpretation of the experiments must be modified to take account of the fact that the activation energy is not independent of temperature as implied by the form of equation (2). Our calculated values of the activation energy for lattice parameters corresponding to room temperature and $1400^{\circ} \mathrm{C}$ differed by $0.3 \mathrm{eV}$. If we assume a linear variation with temperature,

$$
E_{\mathrm{A}}(T)=E_{\mathrm{A}}(0)-\alpha k T,
$$

we have $E_{\mathrm{A}}(0)=2.26 \mathrm{eV}$ and $\alpha=2.46$. Equation (2) may then be written as

$$
\Gamma=\nu_{0} \exp (\alpha) \exp \left(-E_{\mathrm{A}}(0) / k T\right) .
$$

The coefficient $\alpha$ is of course directly related to the activation volume discussed in $\S 2.2$. What is measured is $\Gamma_{0}=\nu_{0} \exp (\alpha)$ but the various theories of jump diffusion give expressions for $\nu_{0}$. For comparison the experimental estimate must therefore be multiplied by a factor $\exp (-\alpha)$, i.e. from experiment $\nu_{0}=18 \pm 7 \mathrm{THz}$. With this reinterpretation, the relevant part of the pre-exponential frequency factor is close to the maximum lattice frequency. It is interesting to note that after this adjustment the experimental estimate is in fair agreement with the estimate of $(3 / 5)^{1 / 2} \nu_{\mathrm{D}}=15.2 \mathrm{THz}$ (taking the Debye temperature for $\mathrm{MgO}$ to be $941 \mathrm{~K}$ ) given by the usual continuum approximation for the dynamical theory (see Flynn 1972). The significant discrepancy between the results of atomistic dynamical theory $(\$ 2.3 .1)$ and the continuum case has already been pointed out. However, the agreement between experiment $(18 \pm 7 \mathrm{THz})$ and Vineyard theory $(32.9 \mathrm{THz})$ can be considered extremely good, since both pre-exponential factor and activation energy are predicted quantities. Our value of $32.9 \mathrm{THz}$ for $\nu_{0}$ calculated from Vineyard theory was obtained from frequencies of the lattice at room temperature. These will also be temperature dependent. With a Grüneisen parameter of unity the expansion of $\mathrm{MgO}$ between room temperature and $1400^{\circ} \mathrm{C}$ would decrease frequencies by $6 \%$. In line with this some detailed calculations which we have made for normal site frequencies show changes of only a few per cent. Therefore we expect the effect produced by any temperature dependence of $\nu_{0}$ to be far less than that of the factor $\exp (\alpha)(\approx 11.7)$ from the temperature dependence of $E_{\mathrm{A}}$. If $\nu_{0}$ is decreased then the agreement between theory and experiment will be improved. We shall see ( $\$ 3.3)$ that the agreement is improved further by going beyond the purely harmonic formulation along the lines discussed in $\S 2.1$.

As a footnote, we would point out that the value which we have assigned to the factor $\exp (\alpha)$ in equation (5) (and which was crucially involved in the reinterpretation of the 
Sempolinski and Kingery (1980) experiments) is open to criticism. A cardinal rule in model calculations such as ours is that all parameters must be determined consistently within the framework of the model. We have violated this by making use of the experimental lattice expansion. We have, however, also estimated the model lattice expansion by the standard method of finding the temperature at which the elastic pressure for a given expansion is balanced by the volume derivative of the vibrational free energy. The expression for the latter involves mode Grüneisen parameters, and these we have approximated by differencing mode frequencies for the expanded and $T=0{ }^{\circ} \mathrm{C}$ lattices for a small sample of modes. We find that for a linear expansion from $a_{0}=2.106 \AA$ to $a_{0}=2.148 \AA$ (being experimental values at $0^{\circ} \mathrm{C}$ and $1400^{\circ} \mathrm{C}$ respectively) the temperature of our model system must be raised from $0^{\circ} \mathrm{C}$ to about $2250^{\circ} \mathrm{C}$. Our overestimate reflects the errors in the elastic constants determined in our potential model (Sangster and Stoneham 1981). This implies that the coefficient $\alpha$ should be reduced from 2.46 to 1.53 and the value for $\nu_{0}$ deduced from experiment would then be $45 \pm 17 \mathrm{THz}$.

\subsection{Corrections to Vineyard theory}

For the Vineyard theory to be valid the energy surface must be strictly harmonic over an energy spread of the order of $k T\left(0.1441 \mathrm{eV}\right.$ for $\left.T=1400^{\circ} \mathrm{C}\right)$. In figure 2 we show our calculated energy changes for displacements of the $\mathrm{Mg}^{2+}$ ion along the $\langle 001\rangle$ directions from the saddle point. The full line represents a polynomial (in the square of the displacement) fitted to our calculations and the broken line is the harmonic term. Neglect of the quartic and higher terms results in a flatter energy surface, and hence the Vineyard theory will overestimate the pre-exponential frequency factor.

If we assume that the only significant departures from harmonicity are those along the $\langle 001\rangle$ directions then the reduction factor which should be applied to the saddle point integral (and hence the pre-exponential frequency factor) is

$$
\int_{0}^{z_{0}} \exp (-E(z) / k T) \mathrm{d} z / \int_{0}^{\infty} \exp \left(-E^{\prime}(z) / k T\right) \mathrm{d} z
$$

where $E(z)$ are our calculated energies and $E^{\prime}(z)$ are the harmonic parts. The upper limit of the integral in the numerator cannot be taken outside the range for which we have carried out calculations and should be sufficiently large that further increases do not significantly increase the integral. We have fitted our calculated energies to a cubic

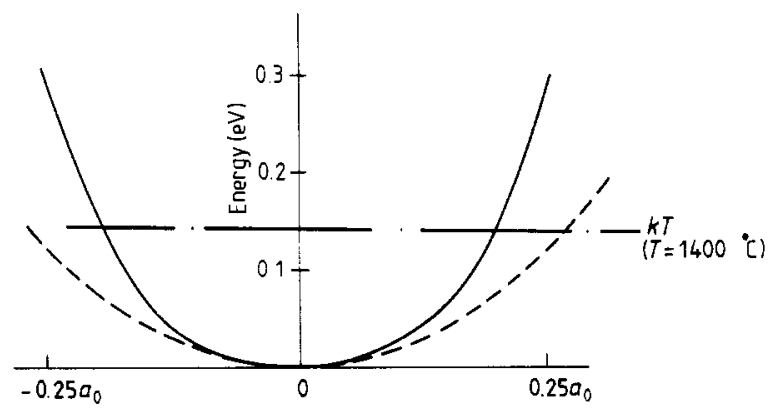

Figure 2. Energy changes for displacements of an $\mathrm{Mg}^{2-}$ ion from the saddle point along the $\langle 001\rangle$ direction. The full line is a polynomial fitted to our calculations; the broken line is the harmonic term. 
polynomial in $z^{2}$ and have taken $z_{0}$ such that $E\left(z_{0}\right)=6 k T$. Increasing the limit to $8 k T$ produces no significant change. (If the integral in the numerator is similarly truncated at $z_{0}$ with $E\left(z_{0}\right)=6 k T$ the value is reduced by a factor of $\left.\operatorname{erf}(\sqrt{ } 6)=0.9995\right)$. Our estimate of the reduction factor using numerical integration for the numerator is 0.70 . The ratio of two-dimensional integrals over the plane shown in figure $1(a)$ (estimated in the same way) is 0.72 which shows that, as is to be expected, the dominant factor arises from the 'soft' direction.

We have not considered the analogous correction factor which should be applied at the equilibrium site. (Since, unlike the saddle point, the equilibrium site does not have inversion symmetry, the polynomial expansion of the energy surface will now include odd powers of the displacement from the site.) This factor could of course be either greater or less than unity. If it is ignored the saddle point correction reduces our calculated value of the pre-exponential frequency factor $(32.9 \mathrm{THz})$ to $23 \mathrm{THz}$, i.e. within the range deduced from experiment $(18 \pm 7 \mathrm{THz})$.

A further reason for our calculated value being slightly too high comes from the assumption of the Vineyard theory that each arrival at the saddle point plane is counted as a crossing, the possibility of a return to the starting configuration being ignored. The question of these 'spurious crossings' has been considered by McCombie and Sachdev (1975) for idealised extremes and more recently by Jacucci et al (1984) for more realistic models. The latter conclude that the return jump fraction is unlikely to exceed $10 \%$.

Our final conclusion is that, by direct calculation using appropriately extended Vineyard theory, agreement with experiment for jump rates can be achieved to an accuracy comparable with experimental accuracy.

\subsection{Experiment and $\mathrm{Fe}^{3+}$ motion}

For the $\mathrm{Fe}^{3-}$ ion, jump our calculations based on Vineyard theory gave a low preexponential frequency factor. Together with the relatively high activation energy for this jump $(2.7 \mathrm{eV})$, this implies a low mobility for the $\mathrm{Fe}^{3+}$ inpurities. In our earlier paper (Sangster et al 1984) we have calculated formation energies for various vacancy-Fe ${ }^{3+}$ pair configurations and activation energies for jumps between them. Several of these energies confirm the view that the $\mathrm{Fe}^{3-}$ impurities are unlikely to be mobile:

(a) The pair with $\langle 100\rangle$ orientation, that is with the vacancy and $\mathrm{Fe}^{3+}$ ion as second neighbours on the cation sublattice, has a binding energy of $0.88 \mathrm{eV}$ and is more stable by $0.27 \mathrm{eV}$ than the $\langle 110\rangle$ pair orientation which is the initial configuration for the jump we have considered. This is in agreement with the energy difference of $0.28 \mathrm{eV}$ found by Gourdin and Kingery (1979). In other words a configuration with a cation vacancy immediately adjacent to an $\mathrm{Fe}^{3+}$ impurity is less favoured than at the second neighbour site.

(b) If a vacancy is found adjacent to an $\mathrm{Fe}^{3+}$ impurity, then $\mathrm{Mg}^{2+}$-vacancy interchanges are more likely than the $\mathrm{Fe}^{3+}$-vacancy interchange. This is partly a matter of lattice geometry, for even in the $\langle 110\rangle$ pair the $\mathrm{Fe}^{3+}$ is only one of the twelve cation neighbours, and partly a matter of energetics, for the activation energies for $\mathrm{Mg}^{2-}$ ion jumps are appreciably smaller than the $2.7 \mathrm{eV}$ required for the $\mathrm{Fe}^{3+}$ jump. The formal theory has been given by Lidiard (1955) (see also Flynn 1972, $\$ \S 6.3,6.4$ ). In figure 3 the four distinct classes into which the $\mathrm{Mg}^{2+}$ ions divide are shown, with the jumps identified to correspond to Lidiard's notation. The activation energy for a jump of an $\mathrm{Mg}^{2+}$ ion of the first class is $2.365 \mathrm{eV}$. Such a jump leaves another $\langle 110\rangle$ vacancy $-\mathrm{Fe}^{3+}$ pair. A jump of an ion of the second class requires an activation energy of $1.96 \mathrm{eV}$ and results in a 


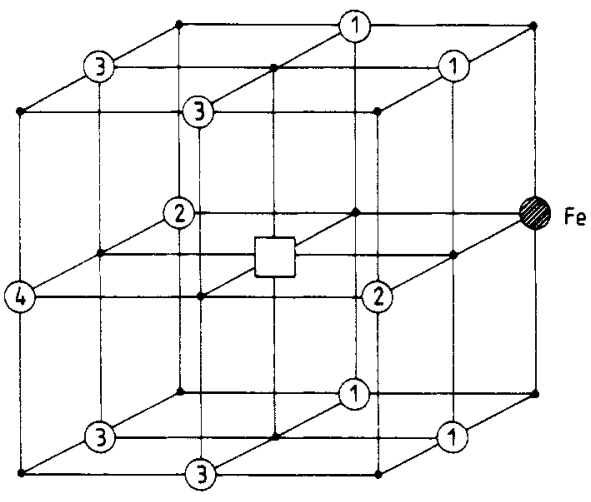

Figure 3. Symmetry classes of $\mathrm{Mg}^{2+}$ near neighbours of an $\mathrm{Fe}^{3+}$-vacancy dimer in $\mathrm{MgO}$. The jump frequencies in the model of Lidiard (1955) can be identified with jumps of ions from specific classes into the vacancy, namely class $1 \equiv w_{1}$, classes $2,3,4$ (all equivalent in the so-called five-frequency model) $\equiv w_{3}:$ the $\mathrm{Fe}^{3-}$ jump $\equiv w_{2}$

$\langle 100\rangle$ dimer. We have not calculated the activation energies for the other two classes, but these will probably lie close to the $2.2 \mathrm{eV}$ required for an $\mathrm{Mg}^{2+}$ ion jump in the absence of $\mathrm{Fe}^{3+}$. From the activation energies alone we conclude that there is a low probability that a vacancy with a nearest-neighbour $\mathrm{Fe}^{3+}$ will exchange sites with the $\mathrm{Fe}^{3+}$ rather than $\mathrm{Mg}^{2+}$ (i.e. $w_{1}, w_{3} \gg w_{2}$ in Lidiard's notation). In this limit, the $\mathrm{Fe}^{3+}$ diffusion constant is simply proportional to the fraction $p_{110}$ of all $\mathrm{Fe}^{3-}$ present as $\langle 110\rangle$ pairs and to the $\mathrm{Fe}^{3+}$-vacancy site exchange frequency $w_{2}$, i.e. $D\left(\mathrm{Fe}^{3+}\right)$ is $p_{110} w_{2} a^{2} / 3$, with $a$ the $\mathrm{Mg}-\mathrm{O}$ spacing. The atomic fraction of $\langle 110\rangle$ pairs is related to the total atomic fraction of iron in a complex way, since there is quite a variety of possible complexes and charge states. Representative values based on experiment are given in figures $8(a, b)$ of Gourdin et al (1979) and $p_{110}$ lies in the range 0.05 to 0.1 .

Experimentally, there are several sets of diffusion data for $\mathrm{Fe}$ in $\mathrm{MgO}$ (see Blank and Pask 1969). However, these present problems in estimating jump frequencies. On the one hand, $\mathrm{Fe}$ is present both as $\mathrm{Fe}^{2+}$ and $\mathrm{Fe}^{3+}$, and assigning the motion to one state or the other cannot be done without ambiguity. On the other hand, the diffusion constant involves a vacancy concentration, and the levels of intrinsic vacancies and of extrinsic vacancies due both to $\mathrm{Fe}$ and to inadvertent impurities are not known. If we use 0.05 for $p_{110}$ and $2 \times 1.21 \times \exp \left(-2.7 \mathrm{eV} / k T\right.$ ) $\mathrm{THz}$ for $w_{2}$ (from $\$ 3.1$ here) we obtain a preexponential factor $D_{0}$ of $1.8 \times 10^{-5} \mathrm{~cm}^{2} \mathrm{~s}^{-1}$, corresponding to a diffusion constant $D$ of only $1.3 \times 10^{-13} \mathrm{~cm}^{2} \mathrm{~s}^{-1}$ at $1400^{\circ} \mathrm{C}$. The full temperature dependence of $D$ will of course include both the $2.7 \mathrm{eV}$ activation energy and the temperature dependence of $p_{110}$. We conclude that isolated $\mathrm{Fe}^{3+}$ contributes negligibly to the observed iron diffusion in $\mathrm{MgO}$. Results are available from other types of experiment, e.g. from aggregation studies (Weeks et al 1980). However, the relative immobility of $\mathrm{Fe}^{3+}$ ions which we predict contrasts with the conclusions which Weeks et al draw from their spin resonance study of the distribution of $\mathrm{Fe}^{3+}$ in doped $\mathrm{MgO}$. Their results are also not in agreement with diffusion rates found in tracer experiments reviewed by Wuensch (1982). It seems probable that the rapid diffusion required to explain the $\mathrm{Fe}^{3+}$ aggregation found by Weeks et al arises from an alternative mechanism such as grain boundary diffusion. 


\section{Conclusions}

Our major conclusion is that successful calculations of absolute diffusion rates are possible with the present generation of computer codes and interatomic potentials. In any such calculations it is important to recognise that quoted experimental pre-exponential factors include a factor $(\exp (\alpha)$ of equation $(6))$ reflecting the change of activation energy with thermal expansion. Furthermore, the normal Vineyard results may need to be modified especially whenever the energy surface near the saddle point (or the equilibrium configuration) is relatively flat.

\section{Acknowledgments}

We are grateful to Dr A B Lidiard and Professor C W McCombie for helpful discussions. The program for supercell lattice dynamics was developed in collaboration with $\mathrm{DK}$ Rowell. This paper is dedicated to the memory of Paul Dean, a long-standing friend and colleague in the solid-state physics community.

\section{References}

Almond D P and West A R 1983 Nature 306456

Blank S L and Pask J A 1969 J. Amer. Ceram. Soc. 52669

Catlow C R A and Norgett M J 1976 AERE Report M2936 Harwell

Evarestov R A and Lovchikov V A 1979 Phys. Status Solidi (b) 93469

Flynn C P 1972 Defects and Diffusion in Solids (London: Oxford University Press)

Gillan M J 1981 Phil. Mag. A 43301

Gillan M J and Jacobs P W M 1983 Phys. Rev. B 28759

Glasstone S, Laidler K J and Eyring H 1941 The Theory of Rate Processes (New York: McGraw-Hill)

Gourdin W H and Kingery W D 1979 J. Mater. Sci. 142053

Gourdin W H, Kingery W D and Driear J 1979 J. Mater. Sci. 142074

Harding J H. Masri P and Stoneham A M 1980 J. Nucl. Mater. 9273

Harding J H and Stoneham A M 1980 Phil. Mag. B 4343

Huberman B A and Boyce J B 1978 Solid State Commun. 25843

Jacobs P W M and Gillan M J 1983 Phys. Rev. B 28759

Jacucci G, Toller M, De Lorenzi G and Flynn C P 1984 Phys. Rev. Lett. 52295

Lidiard A B 1955 Phil. Mag. 461218

__ 1957 Handbuch der Physik 20245 (Berlin: Springer)

McCombie C W and Sachdev M 1975 J. Phys. C: Solid State Phys. 8 L413

Norgett M J 1974 AERE Report R7650 Harwell

1977 AERE Report M2880 Harwell

Page J B and Strauch D 1967 Phys. Status Solidi 24469

Rice S A 1958 Phys. Rev. 121804

Sangster M J L and Rowell D K 1982 J. Phys. C: Solid State Phys. 155153

Sangster M J L, Rowell D K and Ashman A S 1981 J. Phys. C: Solid State Phys. 142889

Sangster M J L and Stoneham A M 1981 Phil. Mag. B 43597

- 1982 Phys. Rev. B 261026

Sangster M J L, Stoneham A M and Rowell D K 1984 Amer. Cer. Soc. in press

Sempolinski D R and Kingery W D $1980 \mathrm{~J}$. Amer. Cer. Soc. 6311

Slater N B 1959 The Theory of Unimolecular Reactions (Ithaca, NY: Cornell University Press)

Vineyard G H 1957 J. Phys. Chem. Solids 3121

Weeks R A, Gastineau J and Sonder E 1980 Phys. Status Solidi (a) 61265

Wuensch J B 1982 Mass Transport in Solids ed. F Beniere and C R A Catlow. (New York and London: Plenum) p 353 